\title{
A Novel Synthesis of Carboxylic Esters from 2-Methyl-4,6-pyrimidyl Dicarbonates and Grignard Reagents
}

\author{
Jae In Lee \\ Department of Chemistry and Plant Resources Research Institute, College of Natural Science, Duksung Women's University, \\ Seoul 132-714, Korea. E-mail: jilee@duksung.ac.kr \\ Received March 22, 2011, Accepted March 29, 2011
}

Key Words : Carboxylic esters, Alkoxycarbonylation, 2-Methyl-4,6-pyrimidyl dicarbonates, Grignard reagents

The carboxylic esters are mostly prepared by the reaction of carboxylic acids or their derivatives with alcohols and a large number of methods have been known. ${ }^{1}$ However, a few methods have been reported on the derivation of carboxylic esters from the alkoxycarbonylation of organometallics via $\sigma$ bond formation between $\alpha$-carbon and carbonyl carbon. Among them, the reaction of alkynyllithiums with methyl chloroformate $^{2}$ or 2,2,2-trichloroethyl chloroformate ${ }^{3}$ affords the corresponding $\alpha, \beta$-alkynoic esters at low temperature, but it requires an excess of chloroformate reagents to avoid competing side reactions. Although the treatment of ethyl chloroformate with organomanganates ${ }^{4}$ or organocopper reagents ${ }^{5}$ associated with magnesium salt under $\mathrm{Pd}$ catalysis produces the corresponding ethyl esters, these organometallics are prepared by an additional step and the scope is limited to primary and alkenyl group, respectively. The direct alkoxycarbonylation of Grignard reagents with dialkyl carbonates $^{6}$ or alkyl chloroformates ${ }^{7}$ affords the corresponding esters, but the former requires 2 equiv of dialkyl carbonates and the latter is limited to the preparation of $\alpha$-diazo esters at $-78^{\circ} \mathrm{C}$. Instead, the alkoxycarbonylation of Grignard reagents proceeds well with 1 equiv of 2-pyridyl carbonates, ${ }^{8}$ but the yield of 2,2,2-trichloroethyl ester depends on reaction temperature.

Recently, alkyl cyanoformates have been utilized for the alkoxycarbonylation of organometallics. Thus, the alkoxycarbonylation of alkynyllithiums ${ }^{9}$ or allenyllithiums ${ }^{10}$ with 2 equiv of methyl or ethyl cyanoformate provides methyl akynoates or ethyl allenates, respectively. The reaction of heteroaromatic magnesium halides with an excess of alkyl cyanoformates also affords carboxylic esters in moderate to high yields. ${ }^{11}$ Furthermore, the treatment of methyl cyanoformate with magnesium metalated nitriles, derived from $\alpha$ bromonitriles and isopropylmagnesium chloride, at $-78^{\circ} \mathrm{C}$ affords methyl $\alpha$-cyanoates in moderate to high yields. ${ }^{12}$

Thus, the success of carboxylic esters synthesis by the alkoxycarbonylation of organometallics depends largely on the nature/amount of alkoxycarbonylating reagents and metals employed. However, there are no reports of alkoxycarbonylating reagents that can produce 2 equiv of carboxylic esters with organometallics. Inspired by our previous reports on ketones synthesis using pyrimidyl diesters, ${ }^{13}$ we investigated the synthesis of carboxylic esters from 2-methyl-4,6- pyrimidyl dicarbonates and Grignard reagents. In choosing 2-methyl-4,6-pyrimidyl group as active moiety, we considered i) chelation effect between nitrogen atom and magnesium atom ii) thermal stability and storage of 2-methyl-4,6-pyrimidyl dicarbonates rather than other pyrimidyl dicarbonates.

2-Methyl-4,6-pyrimidyl dicarbonates 3 were newly prepared by the addition of 2 equiv of alkyl chloroformates 2 to a suspended solution of 4,6-dihydroxy-2-methylpyrimidine 1 and 2 equiv of triethylamine in THF at room temperature (Scheme 1). The nucleophilic acyl substitution of $\mathbf{2}$ with $\mathbf{1}$ was completed in 2-10 h at room temperature because 1 was slightly soluble in THF. After completion of the reaction, the resulting triethylamine hydrochloride precipitate was filtered off. After usual workup, the residue was subjected to silica gel (Davisil, $\mathrm{pH}=7$ ) or recystallized to afford 3 in $67-89 \%$ yields. During the synthesis of 2-methyl-4,6-pyrimidyl diisobutyl dicarbonate 3a, a small amount $(\sim 10 \%)$ of $N, N$ diethyl isobutyl carbamate was produced by the nucleophilic acyl substitution of isobutyl chloroformate with triethylamine, which was distilled out under vacuum. However, 2-

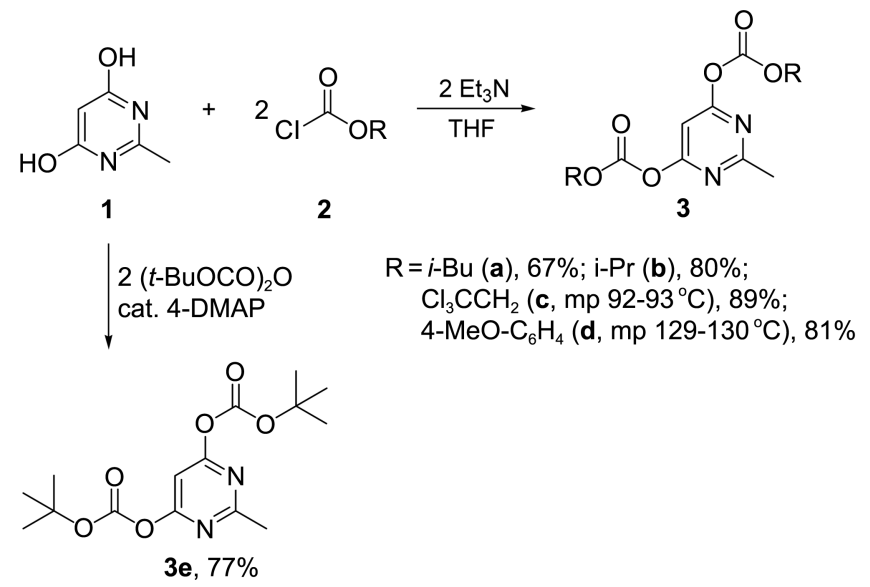

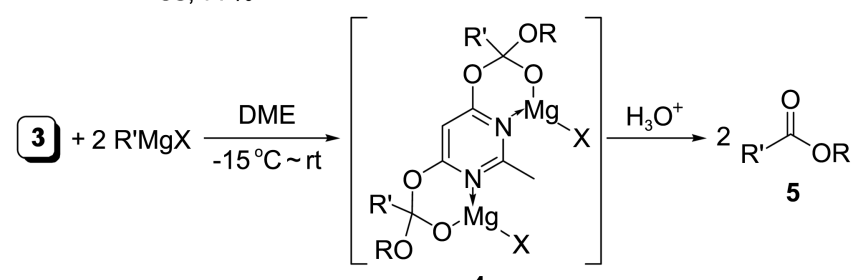

Scheme 1 
methyl-4,6-pyrimidyl di(tert-butyl) dicarbonate $3 \mathbf{e}$ was prepared by the reaction of 1 with 2 equiv of di-tert-butyl dicarbonate in the presence of 0.03 equiv of 4-DMAP in $77 \%$ yield after $8 \mathrm{~h}$ at room temperature because tert-butyl chloroformate was thermally unstable.

The synthesis of carboxylic esters $\mathbf{5}$ was successfully accomplished by the slow addition of 2 equiv of Grignard reagents to a solution of $\mathbf{3}$ in DME. For instance, 2 equiv of $o$-tolylmagnesium chloride was added to a solution of 2methyl-4,6-pyrimidyl diisopropyl dicarbonate $\mathbf{3 b}$ in DME over $5 \mathrm{~min}$ at $-15^{\circ} \mathrm{C}$ and stirred for $2 \mathrm{~h}$ between $-15^{\circ} \mathrm{C}$ and $0{ }^{\circ} \mathrm{C}$. The resulting precipitate was hydrolyzed with saturated $\mathrm{NH}_{4} \mathrm{Cl}$ solution to give isopropyl 2-methylbenzoate $\mathbf{5 f}$ in $81 \%$ yield after usual separation without an appreciable side products. The corresponding reactions in $\mathrm{THF}$ and $\mathrm{Et}_{2} \mathrm{O}$ afforded $\mathbf{5 f}$ in $74 \%$ and $70 \%$ yield, respectively, and thus DME was somewhat more effective for the high yield formation of $\mathbf{5 f}$. The success of alkoxycarbonylation may be ascribed to the formation of 6-membered chelate 4 between magnesium atoms of Grignard reagents and carbonyl oxygen/nitrogen atoms of $\mathbf{3}$, which would react sluggishly with Grignard reagent and was dissociated to give $\mathbf{5}$ after acidic hydrolysis.

As shown in Table 1, various carboxylic esters were efficiently prepared in high yields (72-91\%) by this method. The alkoxycarbonylation of Grignard reagents worked well with both aliphatic and aromatic 2-methyl-4,6-pyrimidyl dicarbonates and the kind of alkyl groups in $\mathbf{3}$ didn't

Table 1. Preparation of carboxylic esters from 2-methyl-4,6-pyrimidyl dicarbonates and Grignard reagents

\begin{tabular}{|c|c|c|c|c|c|}
\hline $\begin{array}{c}\text { Entry } \\
\mathbf{5}\end{array}$ & $\mathrm{R}$ & $\mathrm{R}^{\prime} \mathrm{MgX}$ & $\begin{array}{l}\text { Reaction conditions } \\
\text { temp }\left({ }^{\circ} \mathrm{C}\right) \text {; time }(\mathrm{h})\end{array}$ & $\begin{array}{c}\text { Esters } \\
\text { R'COOR }^{\prime}\end{array}$ & $\begin{array}{l}\text { Isolated } \\
\text { yield, \% }\end{array}$ \\
\hline $\mathbf{a}$ & $\left(\mathrm{CH}_{3}\right)_{2} \mathrm{CHCH}_{2}$ & & $10 \rightarrow \mathrm{rt} ; 1.5$ & & 72 \\
\hline b & & & $-15 ; 0.5$ & & 86 \\
\hline c & $\mathrm{Cl}_{3} \mathrm{CCH}_{2}$ & & $-15 ; 0.5$ & & 82 \\
\hline d & & & $-15 ; 1$ & & 82 \\
\hline e & $\left(\mathrm{CH}_{3}\right)_{2} \mathrm{CH}$ & & $-15 ; 0.5$ & & 76 \\
\hline f & & & $-15 \rightarrow 0 ; 2$ & & 81 \\
\hline g & & & $\mathrm{rt} ; 2$ & & 83 \\
\hline h & $\left(\mathrm{CH}_{3}\right)_{3} \mathrm{C}$ & & $-15 ; 1$ & & 91 \\
\hline $\mathbf{i}$ & & & $0 \rightarrow \mathrm{rt} ; 2$ & & 78 \\
\hline $\mathbf{j}$ & $4-\mathrm{CH}_{3} \mathrm{O}-\mathrm{C}_{6} \mathrm{H}_{4}$ & & $0 ; 1$ & & 80 \\
\hline $\mathbf{k}$ & & & $0 ; 0.5$ & & 84 \\
\hline 1 & & & $0 \rightarrow \mathrm{rt} ; 1$ & & 83 \\
\hline
\end{tabular}


influence on the efficiency of this reaction. Significantly, the reaction of 2-methyl-4,6-pyrimidyl di(2,2,2-trichloroethyl) dicarbonate $3 \mathbf{c}$ and Grignard reagents afforded the corresponding 2,2,2-trichloroethyl esters (5c, 5d) without concomitant substitution of 2,2,2-trichloroethoxy group. Also, the reaction worked well with both aliphatic and aromatic Grignard reagents regardless of the kind of electron donating $(\mathbf{5 b}, \mathbf{5 f}, \mathbf{5 g})$ and electron withdrawing group (5i, 5l) in substituted phenylmagnesium halides. Although the reaction of 3 with phenylethynylmagnesium bromide and 2,4,6-trimethylphenylmagnesium bromide proceeded at room temperature, the corresponding esters $(\mathbf{5 a}, \mathbf{5 g})$ were obtained in $72 \%$ and $83 \%$ yield, respectively.

In conclusion, the present method provides a novel synthesis of 2-methyl-4,6-pyrimidyl dicarbonates and carboxylic esters from 3 and Grignard reagents. It offers some advantages with respects to (i) the preparation of various 2-methyl4,6-pyrimidyl dicarbonates and their thermal stability, (ii) alkoxycabonylations are clean in short times, and (iii) the synthesis of 2 equiv of various carboxylic esters from 1 equiv of $\mathbf{3}$ in high yields.

\section{Experimental Section}

Preparation of 2-Methyl-4,6-pyrimidyl Diisopropyl Dicarbonate 3b (General procedure). To a suspended solution of 4,6-dihydroxy-2-methylpyrimidine (883 mg, 7.0 $\mathrm{mmol})$ in THF $(50 \mathrm{~mL})$ was added triethylamine $(2.05 \mathrm{~mL}$, $14.7 \mathrm{mmol})$ and isopropyl chloroformate $\left(1.0 \mathrm{M}\right.$ in $\mathrm{C}_{6} \mathrm{H}_{5} \mathrm{CH}_{3}$, $14.7 \mathrm{~mL}, 14.7 \mathrm{mmol}$ ) at room temperature. After being stirred for $7 \mathrm{~h}$, the resulting triethylamine hydrochloride was filtered off. The condensed mixture was poured into cold saturated $\mathrm{NaHCO}_{3}$ solution $(50 \mathrm{~mL})$, extracted with dichloromethane $(3 \times 25 \mathrm{~mL})$. The combined organic phases were dried over $\mathrm{MgSO}_{4}$, filtered, and concentrated in vacuo. The residue was purified by short pathway silica gel (Davisil, $\mathrm{pH}=7.0$ ) column chromatography using 30\% EtOAc/nhexane as an eluent to give $\mathbf{3 b}(1.67 \mathrm{~g}, 80 \%)$ as a viscous oil. ${ }^{1} \mathrm{H}$ NMR $\left(300 \mathrm{MHz}, \mathrm{CDCl}_{3}\right) \delta 6.97(\mathrm{~s}, 1 \mathrm{H}), 5.03$ (septet, $J=$ $6.3 \mathrm{~Hz}, 2 \mathrm{H}), 2.68(\mathrm{~s}, 3 \mathrm{H}), 1.39(\mathrm{~d}, J=6.3 \mathrm{~Hz}, 12 \mathrm{H}) ;{ }^{13} \mathrm{C}$ NMR $\left(75 \mathrm{MHz}, \mathrm{CDCl}_{3}\right) \delta 169.4,166.6,150.5,99.5,74.4$, 25.7, 21.6; FT-IR (film) 3097, 2986, 2940, $1772(\mathrm{C}=\mathrm{O})$, 1596, 1389, 1378, 1242, 1091, $911 \mathrm{~cm}^{-1}$; Ms $m / z$ (\%) 153 (61), 126 (100), 110 (31), 85 (25).

Preparation of Isopropyl 2-Methylbenzoate 5 f (General procedure). To a solution of $\mathbf{3 b}(447 \mathrm{mg}, 1.5 \mathrm{mmol})$ in DME $(20 \mathrm{~mL})$ was slowly added $o$-tolylmagnesium chloride $(0.5 \mathrm{M}$ in THF, $6.0 \mathrm{~mL}, 3.0 \mathrm{mmol})$ at $-15^{\circ} \mathrm{C}$. After being stirred for $2 \mathrm{~h}$ between $-15^{\circ} \mathrm{C}$ and $0{ }^{\circ} \mathrm{C}$, the mixture was quenched with saturated $\mathrm{NH}_{4} \mathrm{Cl}$ solution $(5 \mathrm{~mL})$ and the solvent was evaporated in vacuo. The mixture was poured into saturated $\mathrm{NH}_{4} \mathrm{Cl}$ solution $(30 \mathrm{~mL})$, extracted with dichloromethane $(3 \times 20 \mathrm{~mL})$, and washed with saturated $\mathrm{NaHCO}_{3}$ solution $(30 \mathrm{~mL})$. The combined organic phases were dried over $\mathrm{MgSO}_{4}$, filtered, and concentrated in vacuo. The residue was purified by bulb-to-bulb vacuum distillation to give $\mathbf{5 f}(433 \mathrm{mg}, 81 \%)$. ${ }^{1} \mathrm{H} \mathrm{NMR}\left(300 \mathrm{MHz}, \mathrm{CDCl}_{3}\right) \delta$ $7.88(\mathrm{~d}, J=8.1 \mathrm{~Hz}, 1 \mathrm{H}), 7.34-7.37(\mathrm{~m}, 1 \mathrm{H}), 7.20-7.25(\mathrm{~m}$, 2H), 5.24 (septet, $J=6.3 \mathrm{~Hz}, 1 \mathrm{H}), 2.59$ (s, 3H), 1.37 (d, $J=$ $6.3 \mathrm{~Hz}, 6 \mathrm{H}) ;{ }^{13} \mathrm{C} \mathrm{NMR}\left(75 \mathrm{MHz}, \mathrm{CDCl}_{3}\right) \delta 167.3,139.7$, 131.7, 131.6, 130.5, 130.4, 125.6, 68.1, 22.0, 21.7; FT-IR (film) $3026,2980,1715(\mathrm{C}=\mathrm{O}), 1374,1259,1078,738 \mathrm{~cm}^{-1}$; Ms $m / z(\%) 178\left(\mathrm{M}^{+}, 33\right), 136$ (74), 119 (96), 118 (100), 91 (59).

Isobutyl 3-Phenylpropiolate (5a): ${ }^{1} \mathrm{H}$ NMR $(300 \mathrm{MHz}$, $\left.\mathrm{CDCl}_{3}\right) \delta$ 7.57-7.61 (m, 2H), 7.36-7.45 (m, 3H), $4.02(\mathrm{~d}, J=$ $6.7 \mathrm{~Hz}, 2 \mathrm{H}), 2.03$ (septet, $J=6.7 \mathrm{~Hz}, 1 \mathrm{H}), 0.99$ (d, $J=6.7$ $\mathrm{Hz}, 6 \mathrm{H}) ;{ }^{13} \mathrm{C}$ NMR $\left(75 \mathrm{MHz}, \mathrm{CDCl}_{3}\right) \delta 154.3,133.0,130.6$, 128.6, 119.7, 86.1, 80.7, 72.1, 27.7, 19.1; FT-IR (film) 3061, 2964, $2222(\mathrm{C} \equiv \mathrm{C}), 1709(\mathrm{C}=\mathrm{O}), 1490,1376,1283,1191$, 758, $689 \mathrm{~cm}^{-1}$; Ms $m / z$ (\%) $202\left(\mathrm{M}^{+}, 7\right), 146(22), 129$ (100), 102 (77).

Isobutyl 4-Methoxybenzoate (5b): ${ }^{1} \mathrm{H}$ NMR $(300 \mathrm{MHz}$, $\left.\mathrm{CDCl}_{3}\right) \delta 8.01(\mathrm{~d}, J=9.0 \mathrm{~Hz}, 2 \mathrm{H}), 6.92(\mathrm{~d}, J=9.0 \mathrm{~Hz}, 2 \mathrm{H})$, $4.08(\mathrm{~d}, J=6.6 \mathrm{~Hz}, 2 \mathrm{H}), 3.86(\mathrm{~s}, 3 \mathrm{H}), 2.07$ (septet, $J=6.7$ $\mathrm{Hz}, 1 \mathrm{H}), 1.02(\mathrm{~d}, J=6.7 \mathrm{~Hz}, 6 \mathrm{H}) ;{ }^{13} \mathrm{C} \mathrm{NMR}(75 \mathrm{MHz}$, $\left.\mathrm{CDCl}_{3}\right) \delta 166.4,163.3,131.5,123.0,113.6,70.7,55.4,27.9$, 19.2; FT-IR (film) 3107, 2954, 1724 (C=O), 1523, 1416, 1250, 1107, $781 \mathrm{~cm}^{-1}$; Ms $m / z(\%) 208\left(\mathrm{M}^{+}, 12\right), 152$ (80), 235 (100), 107 (15), 92 (17).

2,2,2-Trichloroethyl Phenylacetate (5c): ${ }^{1} \mathrm{H}$ NMR (300 $\left.\mathrm{MHz}, \mathrm{CDCl}_{3}\right) \delta$ 7.27-7.35 (m, 5H), 4.74 (s, 2H), 3.76 (s, 2H); ${ }^{13} \mathrm{C}$ NMR $\left(75 \mathrm{MHz}, \mathrm{CDCl}_{3}\right) \delta 169.9,132.9,129.4,128.6$, 127.4, 94.8, 74.1, 40.9; FT-IR (film) 3032, 2954, 1756 $(\mathrm{C}=\mathrm{O}), 1455,1233,1131,720 \mathrm{~cm}^{-1}$; Ms $m / z(\%) 270\left(\mathrm{M}^{+}+4\right.$, 10), $268\left(\mathrm{M}^{+}+2,38\right), 266\left(\mathrm{M}^{+}, 40\right), 119$ (29), 91 (100).

2,2,2-Trichloroethyl 2-Thiophenecarboxylate (5d): ${ }^{1} \mathrm{H}$ NMR (300 MHz, $\left.\mathrm{CDCl}_{3}\right) \delta 7.94\left(\mathrm{dd}, J_{1}=3.8 \mathrm{~Hz}, J_{2}=1.3\right.$ $\mathrm{Hz}, 1 \mathrm{H}), 7.66\left(\mathrm{dd}, J_{1}=5.0 \mathrm{~Hz}, J_{2}=1.3 \mathrm{~Hz}, 1 \mathrm{H}\right), 7.16\left(\mathrm{dd}, J_{1}\right.$ $\left.=5.0 \mathrm{~Hz}, J_{2}=3.8 \mathrm{~Hz}, 1 \mathrm{H}\right), 4.95(\mathrm{~s}, 2 \mathrm{H}) ;{ }^{13} \mathrm{C} \mathrm{NMR}(75 \mathrm{MHz}$, $\left.\mathrm{CDCl}_{3}\right) \delta 160.4,134.8,133.9,131.9,128.1,94.9,74.2$; FTIR (film) 3107, 2954, 1725 (C=O), 1416, 1250, 1107, 717 $\mathrm{cm}^{-1}$; Ms $m / z(\%) 262\left(\mathrm{M}^{+}+4,10\right), 260\left(\mathrm{M}^{+}+2,32\right), 258\left(\mathrm{M}^{+}\right.$, 32), 112 (16), 111 (100), 83 (13).

Isopropyl Phenylacetate (5e): ${ }^{1} \mathrm{H}$ NMR $(300 \mathrm{MHz}$, $\left.\mathrm{CDCl}_{3}\right) \delta$ 7.23-7.32 (m, 5H), 4.99 (septet, $\left.J=6.3 \mathrm{~Hz}, 1 \mathrm{H}\right)$, 3.57 (s, 2H), 1.22 (d, $J=6.3 \mathrm{~Hz}, 6 \mathrm{H}) ;{ }^{13} \mathrm{C} \mathrm{NMR}(75 \mathrm{MHz}$, $\left.\mathrm{CDCl}_{3}\right) \delta 171.6,134.7,129.6,128.9,127.4,68.6,42.1,22.2$; FT-IR (film) 3031, 2981, $1728(\mathrm{C}=\mathrm{O}), 1497,1374,1263$, 1107, 762, $696 \mathrm{~cm}^{-1}$; Ms m/z (\%) $178\left(\mathrm{M}^{+}, 38\right), 119$ (12), 92 (54), 91 (100).

Isopropyl 2,4,6-Trimethylbenzoate (5g): ${ }^{1} \mathrm{H}$ NMR (300 $\left.\mathrm{MHz}, \mathrm{CDCl}_{3}\right) \delta 6.84$ (s, 2H), 5.29 (septet, $J=6.3 \mathrm{~Hz}, 1 \mathrm{H}$ ), $2.29(\mathrm{~s}, 6 \mathrm{H}), 2.27(\mathrm{~s}, 3 \mathrm{H}), 1.36(\mathrm{~d}, J=6.3 \mathrm{~Hz}, 6 \mathrm{H}) ;{ }^{13} \mathrm{C} \mathrm{NMR}$ $\left(75 \mathrm{MHz}, \mathrm{CDCl}_{3}\right) \delta 169.7,139.0,134.7,131.5,128.3,68.3$, 21.9, 21.1, 19.6; FT-IR (film) 2979, 2924, 1721 (C=O), 1612, 1454, 1374, 1268, 1082, $853 \mathrm{~cm}^{-1}$; Ms $m / z$ (\%) 206 $\left(\mathrm{M}^{+}, 51\right), 164$ (51), 147 (96), 146 (100), 119 (30), 91 (20).

tert-Butyl 3-(1,3-Dioxanyl)propanoate (5h): ${ }^{1} \mathrm{H}$ NMR $\left(300 \mathrm{MHz}, \mathrm{CDCl}_{3}\right) \delta 4.58(\mathrm{t}, J=5.0 \mathrm{~Hz}, 1 \mathrm{H}), 4.09\left(\mathrm{dd}, J_{1}=\right.$ $\left.11.2 \mathrm{~Hz}, J_{2}=5.0 \mathrm{~Hz}, 2 \mathrm{H}\right), 3.70-3.79(\mathrm{~m}, 2 \mathrm{H}), 2.33(\mathrm{t}, J=7.5$ $\mathrm{Hz}, 2 \mathrm{H}), 1.97-2.14(\mathrm{~m}, 1 \mathrm{H}), 1.87\left(\mathrm{td}, J_{1}=7.5 \mathrm{~Hz}, J_{2}=5.0\right.$ $\mathrm{Hz}, 2 \mathrm{H}), 1.44(\mathrm{~s}, 9 \mathrm{H}), 1.30-1.36(\mathrm{~m}, 1 \mathrm{H}) ;{ }^{13} \mathrm{C} \mathrm{NMR}(75$ $\left.\mathrm{MHz}, \mathrm{CDCl}_{3}\right) \delta 172.6,101.0,80.1,66.8,30.4,29.9,28.1$, 
25.8; FT-IR (film) 2974, 2851, 1729 (C=O), 1445, 1367, 1244, $1147 \mathrm{~cm}^{-1}$; Ms $m / z$ (\%) 159 (28), 143 (89), 87 (100), 85 (43), 57 (41).

tert-Butyl 4-Chlorobenzoate (5i): ${ }^{1} \mathrm{H}$ NMR (300 MHz, $\left.\mathrm{CDCl}_{3}\right) \delta 7.92(\mathrm{~d}, J=8.5 \mathrm{~Hz}, 2 \mathrm{H}), 7.38(\mathrm{~d}, J=8.5 \mathrm{~Hz}, 2 \mathrm{H})$, $1.56(\mathrm{~s}, 9 \mathrm{H}) ;{ }^{13} \mathrm{C} \mathrm{NMR}\left(75 \mathrm{MHz}, \mathrm{CDCl}_{3}\right) \delta 164.9,138.8$, 130.8, 130.4, 128.5, 81.4, 28.2; FT-IR (film) 3075, 2979, $1715(\mathrm{C}=\mathrm{O}), 1594,1368,1293,1119,849 \mathrm{~cm}^{-1} ; \mathrm{Ms} m / z(\%)$ $212\left(\mathrm{M}^{+}, 2\right), 157$ (74), 141 (34), 139 (100), 113 (8), 111 (24), 57 (43), 56 (34).

4-Methoxyphenyl 3-Methyl-2-butenoate (5j): ${ }^{1} \mathrm{H}$ NMR $\left(300 \mathrm{MHz}, \mathrm{CDCl}_{3}\right) \delta 7.01(\mathrm{~d}, J=9.0 \mathrm{~Hz}, 2 \mathrm{H}), 6.88(\mathrm{~d}, J=$ $9.0 \mathrm{~Hz}, 2 \mathrm{H}), 5.90(\mathrm{~s}, 1 \mathrm{H}), 3.79(\mathrm{~s}, 3 \mathrm{H}), 2.22(\mathrm{~s}, 3 \mathrm{H}), 1.97$ (s, $3 \mathrm{H}) ;{ }^{13} \mathrm{C}$ NMR $\left(75 \mathrm{MHz}, \mathrm{CDCl}_{3}\right) \delta 165.3,159.6,157.0$, 144.2, 122.5, 115.2, 114.4, 55.6, 27.6, 20.4; FT-IR (KBr) 3023, 2963, $1725(\mathrm{C}=\mathrm{O}), 1509,1200,1132,1030,834 \mathrm{~cm}^{-1}$; Ms $m / z(\%) 206\left(\mathrm{M}^{+}, 23\right), 124$ (91), 109 (25), 83 (100), 55 (31).

4-Methoxyphenyl Cyclohexanecarboxylate (5k): ${ }^{1} \mathrm{H}$ NMR (300 MHz, $\left.\mathrm{CDCl}_{3}\right) \delta 6.97(\mathrm{~d}, J=9.0 \mathrm{~Hz}, 2 \mathrm{H}), 6.87$ (d, $J=9.0 \mathrm{~Hz}, 2 \mathrm{H}), 3.78(\mathrm{~s}, 3 \mathrm{H}), 2.49-2.53(\mathrm{~m}, 1 \mathrm{H}), 2.02-2.06$ $(\mathrm{m}, 2 \mathrm{H}), 1.78-1.82(\mathrm{~m}, 2 \mathrm{H}), 1.52-1.69(\mathrm{~m}, 3 \mathrm{H}), 1.29-1.37$ $(\mathrm{m}, 3 \mathrm{H}) ;{ }^{13} \mathrm{C}$ NMR $\left(75 \mathrm{MHz}, \mathrm{CDCl}_{3}\right) \delta 175.3,157.5,144.8$, 122.7, 114.8, 56.0, 43.6, 29.4, 26.1, 25.8; FT-IR (KBr) 3016, 2931, $1745(\mathrm{C}=\mathrm{O}), 1607,1505,1442,1252,1189,1156$, 1128, $857 \mathrm{~cm}^{-1}$; Ms m/z (\%) $234\left(\mathrm{M}^{+}, 21\right), 124$ (100), 109 (35), 83 (43), 55 (20).

4-Methoxyphenyl 3,5-Dichlorobenzoate (5l): ${ }^{1} \mathrm{H}$ NMR $\left(300 \mathrm{MHz}, \mathrm{CDCl}_{3}\right) \delta 8.05(\mathrm{~d}, J=2.0 \mathrm{~Hz}, 2 \mathrm{H}), 7.61(\mathrm{t}, J=2.0$ $\mathrm{Hz}, 1 \mathrm{H}), 7.11(\mathrm{~d}, J=9.1 \mathrm{~Hz}, 2 \mathrm{H}), 6.94(\mathrm{~d}, J=9.1 \mathrm{~Hz}, 2 \mathrm{H})$, $3.80(\mathrm{~s}, 3 \mathrm{H}) ;{ }^{13} \mathrm{C}$ NMR $\left(75 \mathrm{MHz}, \mathrm{CDCl}_{3}\right) \delta 163.6,158.0$, 144.4, 135.9, 133.7, 132.9, 128.9, 122.6, 115.0, 56.0; FT-IR (KBr) 3066, 2967, 1725 (C=O), 1569, 1508, 1270, 1199, 1032, 880, 815, $752 \mathrm{~cm}^{-1}$; Ms $m / z(\%) 300\left(\mathrm{M}^{+}+4,6\right), 298$ $\left(\mathrm{M}^{+}+2,36\right), 296\left(\mathrm{M}^{+}, 55\right), 175$ (92), 173 (100), 147 (23), 145
(35).

Acknowledgments. This research was supported by the Duksung Women's University Research Grants 3000001215 (2010).

\section{References}

1. (a) Otera, J. Esterification; Wiley-VCH: Weinheim, Germany, 2003; p 5. (b) Buckley, B. R. In Comprehensive Organic Functional Group Transformations II; Katritzky, A. R., Taylor, R. J. K., Eds.; Elsevier: Oxford, U. K. 2005; Vol. 5, p 127.

2. (a) Olomucki, M.; Gall, J.-Y. L.; Barrand, I. J. Chem. Soc., Chem. Commun. 1982, 1290. (b) Mori, K.; Fujiwhara, M. Tetrahedron 1988, 44, 343. (c) Camps, F.; Coll, J.; Llebaria, A.; Moreto, J. M.; Ricart, S. Communications 1989, 123. (d) Reginato, G.; Mordini, A.; Capperucci, A.; Degl'Innocenti, A.; Manganiello, S. Tetrahedron 1998, 54, 10217. (e) Evans, D. A.; Fitch, D. M.; Smith, T. E.; Cee, V. J. J. Am. Chem. Soc. 2000, 122, 10033.

3. Hall, S. E.; Roush, W. R. J. Org. Chem. 1982, 47, 4611.

4. Cahiez, G.; Normant, J. F. Bull. Soc. Chim. France II 1977, 570.

5. Jabri, N.; Alexakis, A.; Normant, J. F. Tetrahedron 1986, 42, 1369.

6. Satyanarayana, G.; Sivaram, S. Synth. Commun. 1990, 20, 3273.

7. Cuevas-Yanez, E.; Muchowski, J. M.; Cruz-Almanza, R. Tetrahedron Lett. 2004, 45, 2417.

8. Lee, J. I.; Kim, S. Bull. Korean Chem. Soc. 1989, 10, 611.

9. Abad, A.; Agullo, C.; Cunat, A. C.; Garcia, A. B.; Gimenez-Saiz, C. Tetrahedron 2003, 59, 9523.

10. Bee, C.; Tius, M. A. Org. Lett. 2003, 5, 1681.

11. (a) Abarbri, M.; Thibonnet, J.; Berillon, L.; Dehmel, F.; Rottlander, M.; Knochel, P. J. Org. Chem. 2000, 65, 4618. (b) Kristensen, J. L.; Veds $\phi$, P.; Begtrup, M. Tetrahedron 2002, 58, 2397. (c) Christophersen, C.; Begtrup, M.; Ebdrup, S.; Petersen, H.; Veds $\phi$, P. J. Org. Chem. 2003, 68, 9513.

12. (a) Fleming, F. F.; Zhang, Z.; Knochel, P. Org. Lett. 2004, 6, 501. (b) Fleming, F. F.; Gudipati, S.; Zhang, Z.; Liu, W.; Steward, O. W. J. Org. Chem. 2005, 70, 3845.

13. (a) Lee, J. I. Bull. Korean Chem. Soc. 2007, 28, 863. (b) Lee, J. I. Bull. Korean Chem. Soc. 2010, 31, 749. 